INPLASY

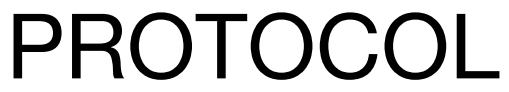

To cite: Gao et al. Omega-3 polyunsaturated fatty acids alter the volume increases of coronary atherosclerotic plaques- a systematic review and meta-analysis. Inplasy protocol 2021110013. doi: 10.37766/inplasy2021.11.0013

Received: 04 November 2021

Published: 04 November 2021

Corresponding author:

Xiaohui Xian

hebmuxxh@hebmu.edu.cn

Author Affiliation:

Department of Pathophysiology, Hebei Key Laboratory of Critical Disease Mechanism and intervention, Hebei Medical University, P.R. China.

Support: There are no funding sources.

Review Stage at time of this submission: Data extraction.

Conflicts of interest:

None declared.

\section{Omega-3 polyunsaturated fatty acids alter the volume increases of coronary atherosclerotic plaques- a systematic review and meta-analysis}

Gao, X1; Zhang, DW2; Yan, XC 3; Shi, HK 4; Xian, XH5.

Review question / Objective: There has been meta-analyses reporting a reduced effect of omega-3 PUFAs trials on cardiovascular events while another two newly publicized meta-analysis indicate that omega-3 PUFAs supplementation had no significant effect on both fatal CHD and any other CHD subtypes. We aimed to draw a more precise conclusion and explain it from a more microscopic physiological perspective. Condition being studied: Omega-3 polyunsaturated fatty acids ( $\omega-3$ PUFAs), one of the two main types of polyunsaturated fatty acids (PUFAs) with the first unsaturated double bond located on the third carbon atom at the methyl end, have been reported to benefit on the cardiovascular system.And the therapeutic efficacy is not easily affected by complications, habits, or interventions. And the American Heart Association AHA has recommended patients diagnosed with coronary heart disease (CHD) to eat about $1 \mathrm{~g} / \mathrm{d}$ EPA +DHA to prevent cardiovascular accidents, while EPA + DHA supplements may should be considered in consultation with physicians. And the reduction of triglycerides in blood is thought to be the key mechanism.

INPLASY registration number: This protocol was registered with the International Platform of Registered Systematic Review and Meta-Analysis Protocols (INPLASY) on 04 November 2021 and was last updated on 04 November 2021 (registration number INPLASY2021110013).

\section{INTRODUCTION}

Review question / Objective: There has been meta-analyses reporting a reduced effect of omega-3 PUFAs trials on cardiovascular events while another two newly publicized meta-analysis indicate that omega-3 PUFAs supplementation had no significant effect on both fatal CHD and any other CHD subtypes. We aimed to draw a more precise conclusion and explain it from a more microscopic physiological perspective. 
Condition being studied: Omega-3 polyunsaturated fatty acids ( $\omega-3$ PUFAs), one of the two main types of polyunsaturated fatty acids (PUFAs) with the first unsaturated double bond located on the third carbon atom at the methyl end, have been reported to benefit on the cardiovascular system.And the therapeutic efficacy is not easily affected by complications, habits, or interventions. And the American Heart Association AHA has recommended patients diagnosed with coronary heart disease (CHD) to eat about $1 \mathrm{~g} / \mathrm{d}$ EPA +DHA to prevent cardiovascular accidents, while EPA + DHA supplements may should be considered in consultation with physicians. And the reduction of triglycerides in blood is thought to be the key mechanism.

\section{METHODS}

Participant or population: Mainly CHD patients or those with very high risk of CHD. Populations of those healthy or with only simple hyperlipidemia and simple diabetics were excluded. The criteria were based on the consensus statement from the European Atherosclerosis Society. And we chose "Per-Protocol analyses" as data for analysis when came across "Intentionto-Treat" and "Per-Protocol analyses". Interventions of newly developed omega-3 PUFA capsules were excluded for the pharmacological uncertainty.

Intervention: We define the supplement of omega-3 PUFAs (such as capsules, combination therapy or just diet) as the interventions. The dose may vary from trials.

Comparator: Placebo or a single drug by stains or other medications.

Study designs to be included: Clinical randomized controlled trials. Intra-group control trials and inter-group control trials.

Eligibility criteria: A retrieval format of PICOS (population, intervention, comparison, outcome, study design) was used to collect study characteristics and outcome data. Three authors (GZ, YXC, and
ZDW) independently extracted outcomes data and recorded the following study characteristics from the included studies: 1 . basic information including publishing year, authors, country, journal; 2. population: age, sex, complications, country, weight, whether or not underwent any heart surgery;3. interventions: the use of placebo, different kinds of $\omega-3$ PUFAs. 4. Outcome: outcome measurement, indicators, time point reported, followingup. 5. Design: randomized controlled trial (RCT) et al. And we collected the differences before and after the follow-up of each group in each outcome measure. One researcher (GZ) transferred data into the Stata 16.1. Another reviewer (ZDW) checked the outcome data again by comparing the data with the study reports to avoid error in data transferring.

Information sources: In August 2021, we searched in Embase statement, Web of science, PubMed, Cochrane Library, Clinical Trial, CNKI and published papers (published from February 1979 to September 2021) for randomized and controlled clinical trials interested in effect of $\omega-3$ PUFAs on coronary atherosclerosis in patients with CHD, without any language restrictions.

Main outcome(s): 1 . reduction in the diameter of the narrowest plaque in the coronary arteries; 2.the volume increases of plaques in coronary arteries.

Quality assessment / Risk of bias analysis: Three of us will independently assesse the performance of each included study in the following quality standards: 1. Random sequence generation 2. Allocation concealment 3 . Blinding of participants and personnel 4. Blinding of outcome assessment 5 . Incomplete outcome data 6 . Selective reporting 7 . Other bias. And we graded each potential source of bias as three levels:" low risk", "unclear risk", and "high risk" under the guidance of the Cochrane Handbook.

Strategy of data synthesis: When studies provide change of outcomes in both 
percentage and absolute value, we will analyze them separately and perform a parallel comparison to make the evidence more deterministic and convincing. The overall effect will be analyzed by "Stata 16.1" (Stata 2020) in a random effect model (Cohen's d). In order to find out the possible source of heterogeneity, sensitivity analyses will be performed using Stata $\mathbf{1 6 . 1}$ for the range of estimates as well as their $95 \%$ confidence interval $(\mathrm{Cl})$. Egger's test and Beggar's test will also be performed by Stata 16.1, which are evaluations of bias in publications.

Subgroup analysis: There is no plan about subgroup analysis.

Sensitivity analysis: In order to find out the possible source of heterogeneity, sensitivity analyses will be performed using Stata $\mathbf{1 6 . 1}$ for the range of estimates as well as their $95 \%$ confidence interval (Cl).

Country(ies) involved: China.

Keywords: omega-3 polyunsaturated fatty acid, coronary heart disease, atherosclerotic plaques.

Contributions of each author:

Author 1 - Zheng Gao.

Email: 2966526665@qq.com

Author 2 - De-Wen Zhang.

Email: 2754301897@qq.com

Author 3 - Xiao-Can Yan.

Email: 1666593916@qq.com

Author 4 - He-Kai Shi.

Email: 2534325742@qq.com

Author 5 - Xiaohui Xian.

Email: hebmuxxh@hebmu.edu.cn 